\title{
LIST EDGE COLORING OF PLANAR GRAPHS WITHOUT 6-CYCLES WITH TWO CHORDS
}

\author{
LINNA $\mathrm{HU}^{a}$, LEI $\mathrm{SUN}^{b}$ \\ AND \\ JIAN-LIANG $\mathrm{WU}^{a, 1}$ \\ ${ }^{a}$ School of Mathematics, Shandong University, Jinan, 250100, China \\ ${ }^{b}$ School of Mathematics, Shandong Normal University, Jinan, 250358, China \\ e-mail: jlwu@sdu.edu.cn.
}

\begin{abstract}
A graph $G$ is edge- $L$-colorable if for a given edge assignment $L=\{L(e)$ : $e \in E(G)\}$, there exists a proper edge-coloring $\varphi$ of $G$ such that $\varphi(e) \in L(e)$ for all $e \in E(G)$. If $G$ is edge- $L$-colorable for every edge assignment $L$ such that $|L(e)| \geq k$ for all $e \in E(G)$, then $G$ is said to be edge- $k$-choosable. In this paper, we prove that if $G$ is a planar graph without 6 -cycles with two chords, then $G$ is edge- $k$-choosable, where $k=\max \{7, \Delta(G)+1\}$, and is edge- $t$-choosable, where $t=\max \{9, \Delta(G)\}$.
\end{abstract}

Keywords: planar graph, edge choosable, list edge chromatic number, chord.

2010 Mathematics Subject Classification: 05C15.

\section{REFERENCES}

[1] M. Bonamy, Planar graphs with $\Delta \geq 8$ are $(\Delta+1)$-edge-choosable, SIAM J. Discrete Math. 29 (2015) 1735-1763.

doi:10.1137/130927449

[2] J.A. Bondy and U.S.R. Murty, Graph Theory with Applications (North-Holland, New York, 1976).

[3] O.V. Borodin, An extension of Kotzig's theorem and the list edge coloring of planar graphs, Mat. Zametki 48 (1990) 22-48.

\footnotetext{
${ }^{1}$ Corresponding author.
} 
[4] O.V. Borodin, A.V. Kostochka and D.R. Woodall, List edge and list total colourings of multigraphs, J. Combin. Theory Ser. B 71 (1997) 184-204.

doi:10.1006/jctb.1997.1780

[5] J.S. Cai, J.F. Hou, X. Zhang and G.Z. Liu, Edge-choosability of planar graphs without non-induced 5-cycles, Inform. Process. Lett. 109 (2009) 343-346. doi:10.1016/j.ipl.2008.12.001

[6] J.S. Cai, List edge coloring of planar graphs without non-induced 6-cycles, Graphs Combin. 31 (2015) 827-832. doi:10.1007/s00373-014-1420-6

[7] F. Galvin, The list chromatic index of a bipartite multigraph, J. Combin. Theory Ser. B 63 (1995) 153-158. doi:10.1006/jctb.1995.1011

[8] R. Hägkvist and J. Janssen, New bounds on the list-chromatic index of the complete graph and other simple graphs, Combin. Probab. Comput. 6 (1997) 295-313. doi:10.1017/S0963548397002927

[9] R. Hägkvist and A. Chetwynd, Some upper bounds on the total and list chromatic numbers of multigraphs, J. Graph Theory 16 (1992) 503-516. doi:10.1002/jgt.3190160510

[10] A.J. Harris, Problems and conjectures in extrema graph theory, Ph.D. Dissertation (Cambridge University, UK, 1984).

[11] J.F. Hou, G.Z. Liu and J.S. Cai, List edge and list total colorings of planar graphs without 4-cycles, Theoret. Comput. Sci. 369 (2006) 250-255. doi:10.1016/j.tcs.2006.08.043

[12] J.F. Hou, G.Z. Liu and J.S. Cai, Edge-choosability of planar graphs without adjacent triangles or without 7-cycles, Discrete Math. 309 (2009) 77-84. doi:10.1016/j.disc.2007.12.046

[13] T.R. Jensen and B. Toft, Graph Coloring Problems (Wiley, New York, 1995).

[14] M. Juvan, B. Mohar and R. Šrekovski, Graphs of degree 4 are 5-choosable, J. Graph Theory 32 (1999) 250-262. doi:10.1002/(SICI)1097-0118(199911)32:3〈250::AID-JGT5〉3.0.CO;2-R

[15] A.V. Kostochka, List edge chromatic number of graphs with large girth, Discrete Math. 101 (1992) 189-201. doi:10.1016/0012-365X(92)90602-C

[16] B. Liu, J.F. Hou and G.Z. Liu, List edge and list total colorings of planar graphs without short cycles, Inform. Process. Lett. 108 (2008) 347-351. doi:10.1016/j.ipl.2008.07.003

[17] Y. Shen, G. Zheng, W. He and Y. Zhao, Structural properties and edge choosability of planar graphs without 4-cycles, Discrete Math. 308 (2008) 5789-5794. doi:10.1016/j.disc.2007.09.048 
[18] W.F. Wang and K.W. Lih, Structural properties and edge choosability of planar graphs without 6-cycles, Combin. Probab. Comput. 10 (2001) 267-276.

[19] W.F. Wang and K.W. Lih, Choosability, edge choosability and total choosability of outerplanar graphs, European J. Combin. 22 (2001) 71-78.

doi:10.1006/eujc.2000.0430

[20] W.F. Wang and K.W. Lih, Choosability and edge choosability of planar graphs without five cycles, Appl. Math. Lett. 15 (2002) 561-565. doi:10.1016/S0893-9659(02)80007-6

[21] J.L. Wu and P. Wang, List-edge and list-total colorings of graphs embedded on hyperbolic surfaces, Discrete Math. 308 (2008) 6210-6215. doi:10.1016/j.disc.2007.11.044

[22] L. Zhang and B. Wu, Edge choosability of planar graphs without small cycles, Discrete Math. 283 (2004) 289-293.

doi:10.1016/j.disc.2004.01.001

Received 22 May 2017

Revised 10 September 2018

Accepted 10 September 2018 